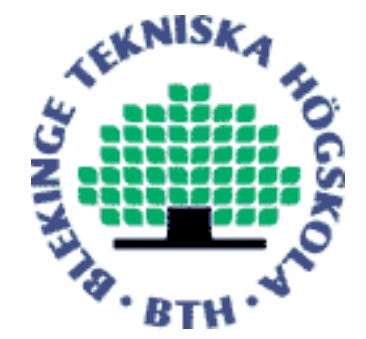

Copyright (C) 2010 IEEE.

Citation for the published paper:

Agile Practices in Global Software Engineering - A Systematic Map

Samireh Jalali, Claes Wohlin

International Conference on Global Software Engineering (ICGSE)

2010 Princeton, NJ

This material is posted here with permission of the IEEE. Such permission of the IEEE does not in any way imply IEEE endorsement of any of BTH's products or services Internal or personal use of this material is permitted. However, permission to reprint/republish this material for advertising or promotional purposes or for creating new collective works for resale or redistribution must be obtained from the IEEE by sending a blank email message to pubs-permissions@iee.org.

By choosing to view this document, you agree to all provisions of the copyright laws protecting it. 


\section{Agile Practices in Global Software Engineering - A Systematic Map}

\author{
Samireh Jalali \\ Blekinge Institute of Technology \\ SE-372 25 Ronneby, Sweden \\ samireh.jalali@bth.se
}

\author{
Claes Wohlin \\ Blekinge Institute of Technology \\ SE-372 25 Ronneby, Sweden \\ claes.wohlin@bth.se
}

\begin{abstract}
This paper presents the results of systematically reviewing the current research literature on the use of agile practices and lean software development in global software engineering (GSE). The primary purpose is to highlight under which circumstances they have been applied efficiently. Some common terms related to agile practices (e.g. scrum, extreme programming) were considered in formulating the search strings, along with a number of alternatives for GSE such as offshoring, outsourcing, and virtual teams. The results were limited to peer-reviewed conference papers/journal articles, published between 1999 and 2009. The synthesis was made through classifying the papers into different categories (e.g. research type, distribution). The analysis revealed that in most cases agile practices were modified with respect to the context and situational requirements. This indicates the need for future research on how to integrate all experiences and practices in a way to assist practitioners when setting up noncollocated agile projects.
\end{abstract}

Keywords-systematic map, agile, scrum, lean software development, extreme programming, pair programming, global software engineering, global software development, offshore, outsource, virtual team, distributed team, open source.

\section{INTRODUCTION}

Distributed teams consisting of stakeholders from different national and organizational cultures, different geographic locations and potentially different time zones characterize global software engineering. These characteristics have significant effects on communication, coordination, and control, and mitigating the effects is a challenge [16].

In comparison with plan-driven software development approaches, agile methods are more flexible when it comes to taking requirements' changes into consideration in all phases of software development [7]. They emphasize extensive collaboration between customers and developers, and encourage small self-organized collocated teams [12].

Although mitigating the GSE challenges by themselves is not a straightforward task, combining agile practices with a global or distributed context complicates things even further. Frequent face-to-face communication among collocated team members improves a feeling of "teamness" and builds trust [5], whilst distance in GSE implies a different way of working, organizational standards, organizational cultures and policies, which may decrease a team's cohesion.

However, (globally) distributed agile has attracted attention due to its potential associated benefits such as shorter time to market, reduced development cost, and managing late requirements' changes. This indicates the need for investigating the experiences reported in the current research literature to determine how agile practices can be efficiently applied in (globally) distributed projects. Although several studies have reported successful integration of agile and GSE (e.g. [S77][S14]), a thorough analysis of the studies to reveal the applicability of the reported experiences and best practices in different organizational settings and project demands is yet unexplored.

The objective of this study is to first summarize the current research literature, and then to investigate which agile practices have been used effectively in a GSE context. Hence, a systematic review was conducted limited to peerreviewed conference papers or journal articles, published between 1999 and 2009.

The remainder of the paper is organized as follows. Section 2 gives a brief background and summarizes the related work. Section 3 discusses the research methodology and explains different steps of conducting this systematic map. The results of the study are presented in Section 4, and finally conclusions and future research directions are presented in Section 5.

\section{BACKGROUND AND RELATED WORK}

The agile practices and GSE alternatives are shortly presented in this section following by putting agile practices in the context of GSE. Moreover, related research work regarding agile practices and GSE is summarized, and finally the motivations and objectives of this study are explained.

\section{A. Agile Practices}

The agile software development approach aims at overcoming the limitations of plan-driven approaches through considering changes of the system's requirements [7]. Agile methods focus on establishing close collaboration between customers and developers, and delivering software within time and budget constraints. Since they rely on frequent informal face-to-face communication rather than providing lengthy documentation, the process is repetitive, adaptive, and minimally defined [4].

The key features of agile methods are continuous requirements gathering; frequent face-to-face communication; pair programming; refactoring; continuous integration; early expert customer feedback; and minimal documentation [S6]. The most widely used methodologies based on the agile principals are extreme programming (XP) and Scrum. However, other methods such as feature-driven development and the crystal clear method have been also used [1].

\section{B. Global Software Engineering}

Geographically distributed software development teams characterize distributed software development, whilst 
globally distributed teams characterize global software development [11]. In this study, we have considered both as GSE. The description of different terms related to GSE is inspired by [11], and the authors have only made minor changes and generalization presented as follows.

Outsourcing (offshore/onshore outsourcing): an external company is responsible for providing software development services or products for the client company. When both subcontracting and client companies are located in the same country, it is known as onshore outsourcing.

Offshoring (offshore insourcing): a company creates its own software development centers located in different countries to handle the internal demand.

Distributed team: team members are spread in different locations and work remotely on different parts of the project (independent tasks) with or without any face-to-face interactions. The difference between a virtual and a distributed team is that virtual team members work jointly on the same tasks.

\section{Agile Practices in Global Software Engineering}

Although agile methods are well suited when customers and developers are collocated and there is frequent interaction among them [3], several software organizations have reported their successful experience of incorporating agile in distributed software development (e.g. [S77][S14]). However, there are challenges associated with this combination, and to get it to work effectively considerable effort is needed. The major difficulties are summarized as related to communication, personnel, culture, different time zones, trust, and knowledge management [S6]. Nevertheless, various tactics and solutions are also reported by different software organizations to mitigate these challenges.

\section{Related Work}

Here, a summary of the previous relevant research is presented. Systematic review studies on agile methods or/and global software engineering are briefly presented. In addition, studies that have partially explored the combination of any agile method in any GSE context are introduced even though if they are not a systematic review study.

Dybå and Dingsøyr [6] have conducted a systematic review of empirical studies of agile software development up to 2005 resulted in identifying 36 relevant empirical studies. Besides the comprehensive analysis of the papers, the need to increase both the number and the quality of studies and to establish a common research agenda in the area of study is pinpointed.

In a systematic review study by Smite et al. [14] the empirical evidence in GSE-related research literature has been investigated. The amount of empirical studies in the area was found to be relatively small, hence it is concluded that the GSE field is still immature. Hence, they have shed light on paths for future work for both researchers and practitioners.

Taylor et al. [S65] conducted a study in 2006 to evaluate the usefulness for practitioners of the existing research on agile global software development. The study included articles published between 2001 and 2005. They concluded that the published research is of minimal value to practitioners since they do not provide novel guidance particularly for distributed agile. It is concluded that the current research of experience reports is similar to the guides available before introduction of agile.

Bose [S6] performed an interesting study in 2008. He selected 12 case studies from literature that claimed to be successful in distributed agile software development, and summarized them. The cases were evaluated in comparison with the agile manifesto to determine to what extent agile values and principles are followed. He discovered some innovative reported solutions for overcoming the challenges of distributed agile development. The conclusion was that although many solutions seemed to be unique for the context of the challenges, they can still suitably guide companies in establishing and running distributed agile software development.

Paasivaara et al. [S44] have described how Scrum practices were adopted to benefit from distributed software development. Multiple case studies were conducted and the collected lessons learned were summarized. In addition, they have summarized the results of literature review on practices used in distributed agile software development. However, the main contribution is not to explore the previous work. Hence, a systematic literature review has not been conducted.

The only systematic literature review in the area is published in 2009, and is performed by Hossain et al. [S27]. It reviews 20 primary papers and identifies challenges of using Scrum in global software development. Additionally, the best practices addressing the identified challenges have been extracted. The presented guidelines and conclusions can help both practitioners and researchers in the area.

\section{E. Motivations and Objectives}

Confirming the findings of the previous works [14][S27], the existing research in the area is exploratory in nature and mostly reports the cases in which some challenges were faced and some strategies were applied. It is also confirmed that lessons learned in one context may not directly apply in another one [13]. Hence, a standard approach for applying agile in GSE does not exist.

Exploring previous research showed that a comprehensive systematic review that covers all agile methods in all GSE settings does not yet exist. Such a systematic review helps identifying different conditions and factors, which affect the success of agile methods in GSE contexts. Hence, this study aims at systematically reviewing and summarizing the existing research literature, and investigating which agile practices have been used effectively in a GSE context. The results and findings may help practitioners in visualizing the risks and benefits of agile global software development, and hence improving the performance in their work. It also helps researchers in obtaining an overview of the status of the area and highlighting the gaps. 


\section{RESEARCH METHOD AND CONDUCT}

The research was initially designed to be a systematic literature review following the guidelines provided by Kitchenham and Charters [8]. The first phase of the study was to draw a systematic map, in which the guidelines on how to conduct a systematic review was considered along with guidelines provided for performing a systematic map by Petersen et al. [9]. This report presents all steps taken in designing and conducting the systematic review, and presents the results considering the systematic map guidelines.

\section{A. Research Questions}

Regarding the need for conducting a systematic literature review in the area, the research questions for this study set to be as follows.

1) RQ.1. What is reported in the current peer-reviewed research literature about Agile practices in GSE?: In order to answer this question, the current research literature had to be explored.

2) RQ.2. Which agile practices, in which GSE settings, under which circumstances have been successfully applied?: To answer this question, the results of the systemaric review had to be synthesized comprehensively.

\section{B. Search Strategy}

The research started with defining a suitable scope, which was initially set to cover all agile practices in all types of distributed development. It led to setting the preliminary research questions, and identifying the keywords. The initial keywords were searched in well-known databases such as ACM Portal and IEEE Xplore. Based on the search results, the research scope, research questions, and keywords were refined, search strings were reformulated, and searches were re-conducted. Moreover, the list of databases was expanded to collect as many relevant papers as possible. In parallel, a list of key papers was generated, which was used as a validation list to ensure the reliability and relevancy of the searches and to evaluate the search strings. The summary of the process is shown in Figure 1.

\section{Data Sources}

In a progressive process as discussed previously, the databases were decided as follows.

1) ACM Portal (http://portal.acm.org): This provides a collection of citations and full-text from ACM journal and newsletter articles and conference proceedings and covers IT and programming areas.

2) IEEE Xplore (http://ieeexplore.ieee.org): It covers electrical engineering, computer science, and electronic subject areas, and provides full-text and bibliographic access to IEEE transactions, journals, magazines and conference proceedings published since 1988.

3) Inspec (http://www.engineeringvillage2.org): Inspec gives bibliographic access to scientific literature in several subjects including electrical engineering, control engineering, information technology, communications, computers, and computing. It is an online database that includes literature from 1969 to the present, and records are updated weekly.

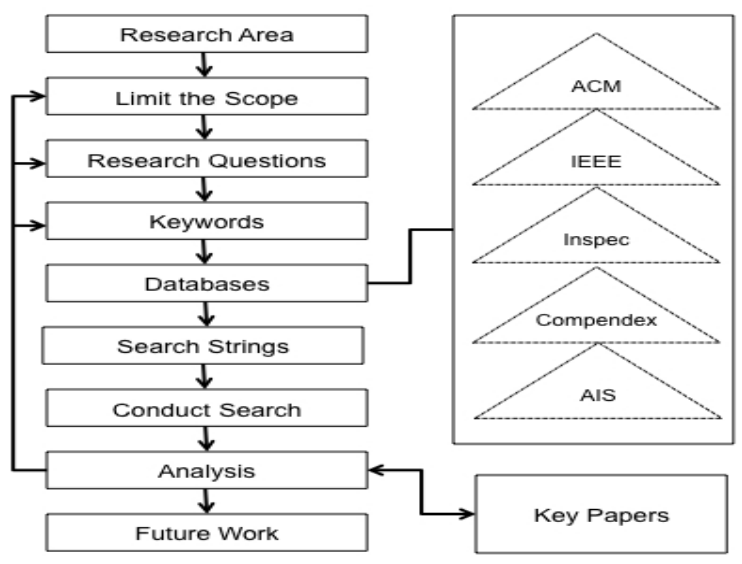

Figure 1. Search strategy and process

4) Compendex (http://www.engineeringvillage2.org): Compendex is a comprehensive interdisciplinary engineering database with 9 million records referencing 5,000 engineering journals and conference materials since 1884 .

5) AIS (http://aisel.aisnet.org): It covers research papers and journal articles in information systems (IS). The reason for including AIS elibrary was to cover papers with an IS perspective.

\section{Data Retrieval}

Search strings were formulated by combining different agile practices and different types of distribution. It can be summarized as: (X1 OR X2 ... OR Xn) AND (Y1 OR Y2 ... OR Yn), where $X$ covers most common agile practices and $Y$ includes different alternatives of GSE as presented in the following.

X: agile, scrum, extreme programming, pair programming, lean development, lean software development

Y: \{global software engineering, global software development, distributed software engineering, distributed software development, GSE, GSD, distributed team, global team, dispersed team, spread team, virtual team, offshore, outsource, open source\}

Agile practices were limited to scrum, extreme programming, pair programming, and lean software development, intending to cover the most common ones, which are mostly used in practice. In addition, the objective was to ensure a clear focus on the scope of the systematic review. However, all spelling alternatives of keywords were considered (e.g. offshore, offshoring, off-shore, offshored, etc).

Furthermore, some limitations were applied on the searches. The publication year was set to be between 1999 and 2009 with the purpose of summarizing the updated relevant related work in approximately the past decade. The written language was set to be English. In order to reduce the 
number of irrelevant hits, the search places were limited to title, abstract, and keywords. It should be noted that only peer-reviewed publications were taken into consideration and gray literature has not been explored.

\section{E. Inclusion Process}

The steps taken to extract the final set of studies for further synthesis are summarized in Figure 2. The searches resulted in identifying 192 papers. The decision on inclusion/exclusion criteria was made based only on the abstract due to the fact that the full-text was not available for many of the papers. This was due to the fact that it was deemed very difficult to order and pay for the papers and then later find out that they should be removed from the further analysis. Based on the evidence found in the title, abstract or keywords implicitly or explicitly, the papers were categorized as "relevant", "irrelevant" or "maybe relevant".

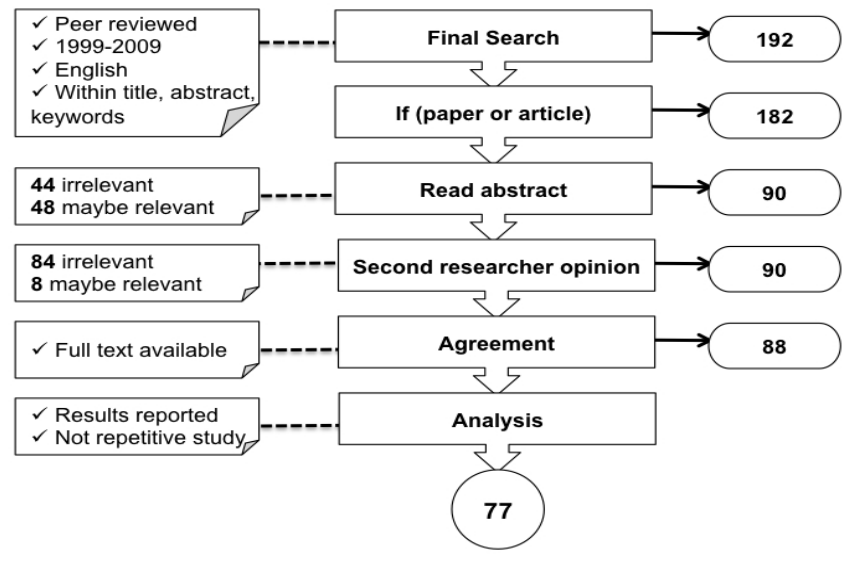

Figure 2. Inclusion process and results

In order to decrease the single researcher's bias at this stage, the list of "irrelevant" and "maybe relevant" ones was given to the second researcher without showing the previous judgments. The result of the second judgment was slightly different regarding the "irrelevant" papers. However, it was decided not to include the papers with one "irrelevant" vote and one "maybe relevant". Papers that both researchers classified them as "maybe relevant" were included in the further analysis.

Finally, both researchers agreed upon a final set of papers for in-depth analysis. If the full paper was not accessible, an email was delivered to the main or second author asking for the paper in pdf. At the analysis step of this study, two emails remained unanswered, so those two papers were excluded. In addition, papers with no result or the same content as other studies were excluded. Thus, 77 studies were finally selected as primary papers for data extraction and synthesis.

\section{F. Data Extraction and Synthesis}

The guidelines provided by Petersen et al. [9] were used to build the classification scheme. Although they have suggested exploring the text adaptively if the abstract was not well structured, we decided to study full-text. We piloted a few studies and realized that critical information such as agile practices, distribution type, and research method could not be extracted only from the abstract.

MS Excel was used for data extraction and collection (see Appendix 1). The items in the form were selected in alignment with the objectives of this study aiming at enabling the authors to answer the research questions by analyzing the extracted data.

All 88 papers were fully read and 11 were excluded at this stage because either the results were not reported or the same study was reported more than once. Hence, data analysis was made for 77 remained papers, and the required items were extracted, coded, and stored in Excel sheets. Finally, several descriptive classifications of the content of the studied papers were made with respect to research methodology, empirical background, findings, participants, and context of the studies.

\section{RESULTS}

The data required for analysis was extracted by exploring the full-text of each included paper. This section presents the collected data.

\section{A. Results of Literature Review}

The outcome of the selection phase was 77 peerreviewed papers and articles. Table I shows the number of papers for each studied year (1999-2009). The maximum was in 2008 with 20 papers, and no relevant paper was found in 1999, 2000, and 2001 as well as few papers in 2002 and 2003. This seems to indicate that GSE and agile in combination has received more attention in the last five years. This is not surprising given that the interest for both agile and GSE have increased during the last 5-10 years.

TABLE I. DISTRIBUTION OF PAPERS OVER THE STUDIED YEARS

\begin{tabular}{|c|c|c|c|c|c|c|c|c|c|c|c|}
\hline & 亏ू & ஓి & ฮ్తి & 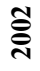 & હ્ఠ & ఫ্ণ & §̊̊ & ఫ્ণ & 尺े & 吕 & હે \\
\hline ACM & & & & & & 2 & 2 & 3 & & & 2 \\
\hline IEEE & & & & & 1 & 2 & 1 & 2 & 6 & 15 & 9 \\
\hline Compendex & & & & 1 & & 1 & 2 & 4 & 4 & 2 & 2 \\
\hline Inspec & & & & & 1 & 5 & 1 & 3 & 2 & 1 & \\
\hline AIS & & & & & & & & & 1 & 2 & \\
\hline Total & $\mathbf{0}$ & $\mathbf{0}$ & $\mathbf{0}$ & 1 & 2 & 10 & 6 & 12 & 13 & 20 & 13 \\
\hline
\end{tabular}

The classification scheme suggested by Wieringa et al. [15] was used as a basis for determining the research type for the set of papers. A short description of each category, which was considered in this study, is provided below.

1) Evaluation Research: Techniques or solutions are implemented and evaluated in practice, and the consequences are investigated.

2) Validation Research: Techniques are novel, but still have not been implemented in practice. This is typically a study of a technique in a laboratory environment.

3) Solution Proposal: A solution for a problem is proposed, and the benefits are discussed. The difference between a solution proposal and a validation research is in the level of abstraction for suggested solutions, which is higher for solution proposals. 
4) Philosophical Paper: It structures the area in the form of a taxonomy or conceptual framework, hence sketches a new way of looking at existing things.

5) Experience paper: It includes the personal experience of the author on what and how something happened in practice.

6) Opinion Paper: The personal opinion on a special matter is reflected in an opinion paper without relying on related work and research methodologies.

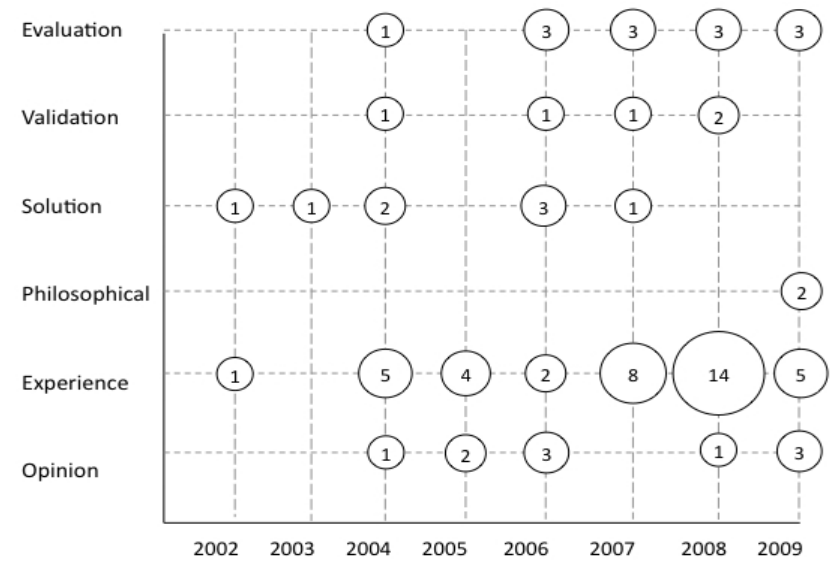

Figure 3. Distribution of research types over the studied years

The results of the categorization are presented in Figure 3. It shows that the majority of the current literature is in the form of experience reports, in which practitioners have reported their own experiences on a particular issue and the method used to mitigate it. The distribution of different research types over studied years pinpoints the need for conducting more philosophical, validation, and evaluation researches. Although experience reports are valuable, evaluation and validation researches with rigorous research method are required to establish foundations for a more mature area.

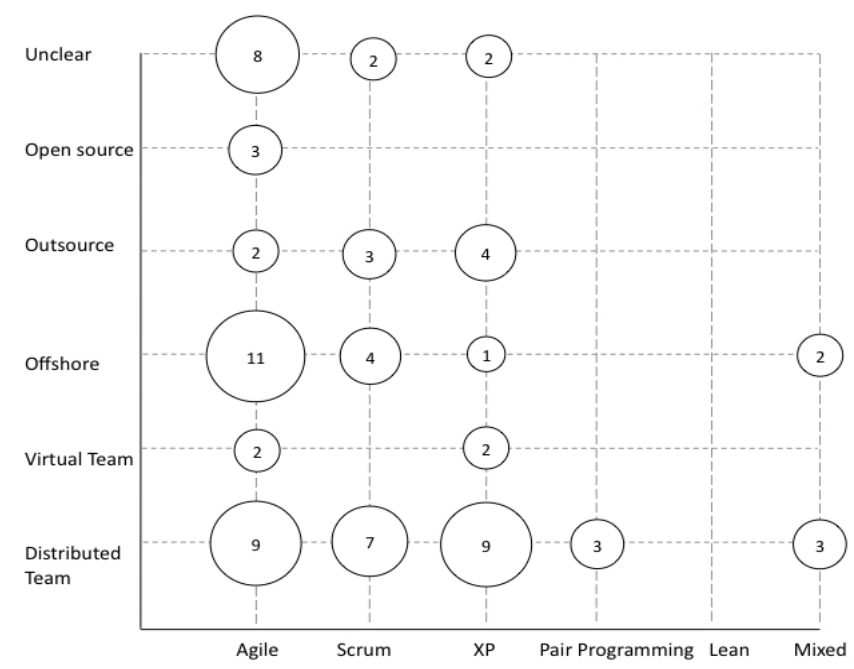

Figure 4. Mapping agile practices and distribution types
Furthermore, the collected data was processed to check which agile practices had been applied in which distribution settings (see Figure 4). The current literature is mostly claiming at applying "agile" as a general term, and the "distributed team" seems to be the most used team/organization setting in GSE collaborations. However, 12 studies did not report the context, and it was not derivable from the full-text of their studies. The lack of context and the quite general formulations regarding agile and team make it difficult for others to make use of the findings.

\section{B. Successful Applications}

Among all included papers, 60 of them were empirical studies: 38 papers were written by practitioners; 19 were written by academic researchers; and three joint papers between practitioners and academia. As shown in Figure 5, 49.5 success stories were reported in the literature. If a report discussed $\mathrm{N}$ projects, the success/failure number for each of them was counted as $1 / \mathrm{N}$.

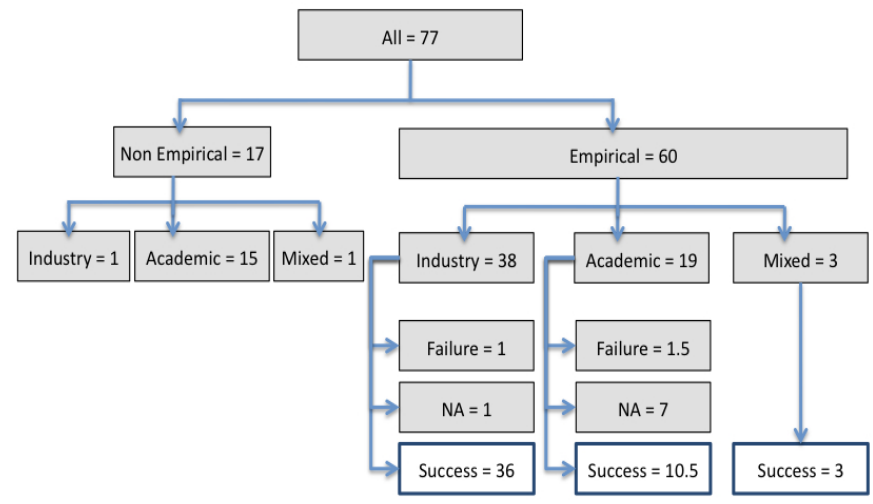

Figure 5. Number of sucessful empirical studies

The most used combination of agile methods and distribution settings are agile-distributed teams, agileoffshore, and XP-distributed teams. Vague use of "agile" and "distributed team" indicates the incompleteness of the contextual and background information in the current literature.

All successful cases were explored in order to identify the applied agile practices. The practices and their frequencies are summarized in Figure 6. According to the available research literature, continuous integration and daily standup scrum meetings are the two activities, which are efficiently practiced the most.

Although several practices were reported in the literature, in many cases it was unclear which agile method has been particularly used. It was also observed that some cases claimed to be agile while too few practices were actually used. Hence, the reliability of their findings cannot be ensured. As a consequence, extra caution is required when using their best practices. The papers were also analyzed to extract the reported projects' main characteristics such as size, duration, domain, and the knowledge area. 


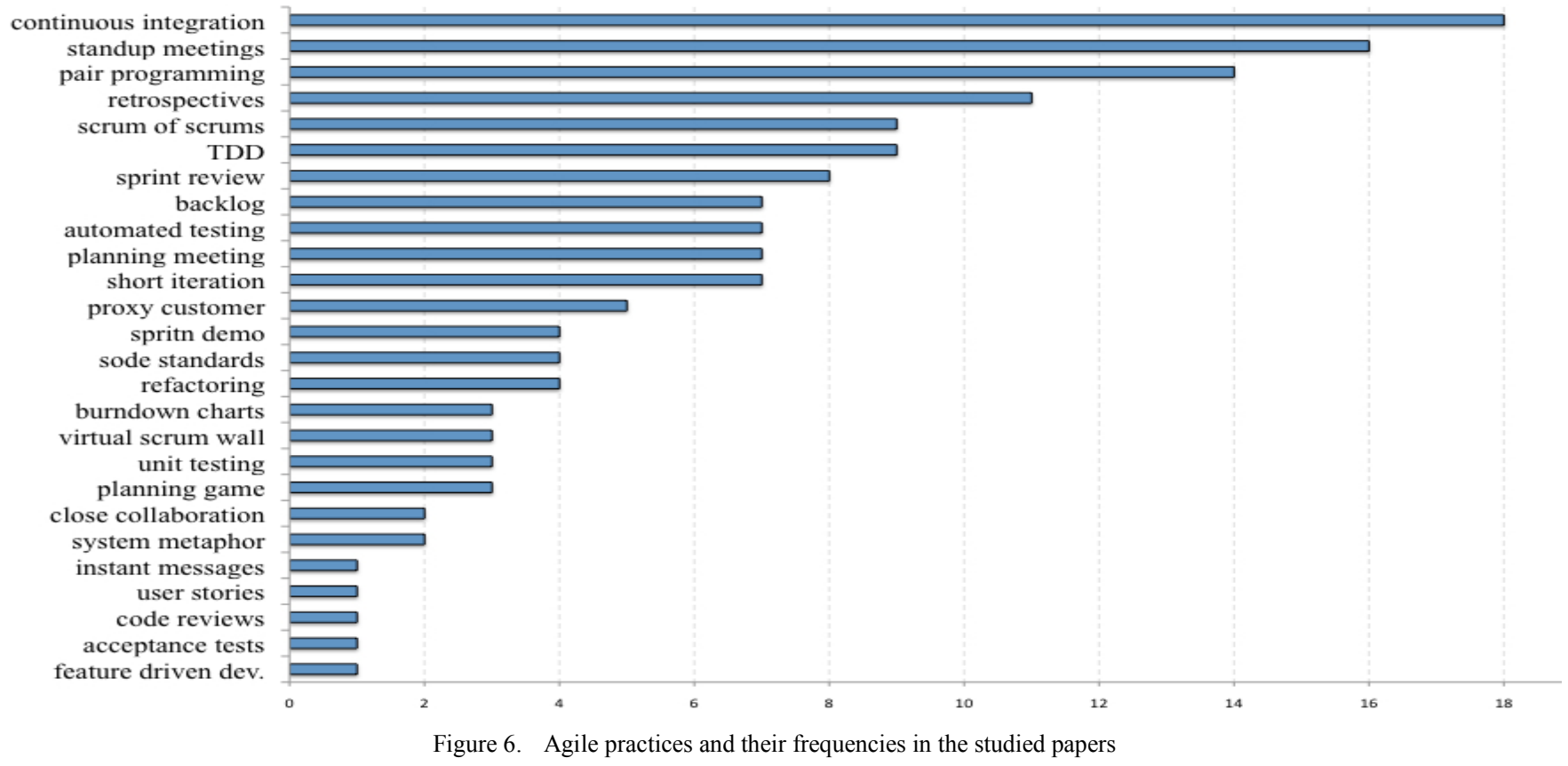

In most cases, the team was distributed around the globe, working for a long time period on a small to medium size project. This can be concluded from Table II. The project size was judged based on the following assumption: Small $<=20$-person $<$ Medium $<=50$-person $<$ Large.

TABLE II. SUMmary OF STUdied PAPERS' FeATURes

\begin{tabular}{|c|c|c|c|}
\hline Distribution & & Global? & \\
\hline Distributed team & |||||||||||||||||||||||||||| & Yes & |||||||||||||||||||||||||||||||||||||||||| \\
\hline Offshore & |||||||||||| $\mid$ & No & |: \\
\hline Outsource & |||||| $\mid$ & Unclear & ||||||||||||||$:$ \\
\hline Virtual team & I & & \\
\hline Open source & I & & \\
\hline Unclear & |l| & & \\
\hline Duration & & Size & \\
\hline Long & ||||||||||| & Large & |||||| \\
\hline Medium & |||||| & Medium & |||||| $\mid$ \\
\hline Short & & Small & $|\||||||||:$ \\
\hline Unclear & 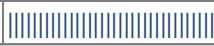 & Unclear & ||||||||||||||||||$:$ \\
\hline Knowledge Area & & Domain & \\
\hline Requirement & $\|:$ & Web & |||||||| $\mid$ \\
\hline Design & $\|$ & Automotive & 1 \\
\hline Construction & $\||||| \mid$ & Service & 1 \\
\hline Testing & ||l| & Embedded & 1 \\
\hline SE Management & |||||| $\mid$ & Telecom & || $\mid$ \\
\hline SE Process & \|\|$\|$ & Real time & 1 \\
\hline Maintenance & \|\|$\|$ & Commercial & 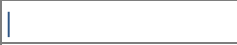 \\
\hline Tools \& methods & \|\|$\|$ & Business critical & \\
\hline \multirow[t]{2}{*}{ Unclear } & |||||||||||||||||| $\mid$ & Finance & I \\
\hline & & Unclear & |||||||||||||||||||||||||||||||||||||||| $\mid$ \\
\hline
\end{tabular}

The duration was considered short if it was less than one month, and long if longer than 7 months. The specification of knowledge areas is based on SWEBOK [2]. The context and project/organizational setting (e.g. application domain, knowledge area, duration, size) were not well documented in most studies. This is shown by a large number of studies being classified as "Unclear" in Table II.

\section{Summary}

Summarizing the relevant research literature provided the answer to the RQ.1. The experience reports of working with globally distributed teams constitute the major part of the literature. They have contributed by explaining the issues, specific solutions, and the lessons learned. However, the majority of them have not documented the characteristics of their empirical study and the context under which the project was running.

The success reports were examined to find the answer to the RQ.2. The existing literature mainly consists of successful empirical experiences (see Figure 5), in which globally distributed teams collaborate over a long time on small to medium sized projects (Figure 4, Table II). Several practices were found in the literature, which have been applied in software organizations. The most common practices used according to the literature are continuous integration, daily standup scrum meetings, pair programming, retrospectives, scrum of scrums meetings, and test-driven development (TDD).

\section{Limitation}

The major concern with any type of research is the reliability. Therefore, two researchers were involved in this systematic map study, discussing the reliability threats early in the design phase. The procedure was discussed and agreed considering the activities to mitigate the effect of one researcher's bias.

The results of the searches were judged for inclusion/exclusion jointly as discussed in Section 3. The co- 
researcher reviewed one random paper, which was previously reviewed by the leading researcher of this study. The purpose was to measure the differences between the results of their data extraction, aiming at minimizing the bias and increasing the accuracy in data collection and categorization.

In order to address the conclusion validity, we collected as many papers as possible from a variety of sources including ACM, IEEE, AIS, Inspec, and Compendex online catalogs. Although different disciplines use different terminologies (e.g. for distributed team), we included as many alternatives as possible for the keywords when formulating the search strings. In addition, the publication year was set to be from 1999 to 2009, which was wide enough to capture most of the relevant publications due to the fact that common agile practices are not much older than one decade. So, it was possible to observe the trends in the area over the past decade.

However, replicating this study may result in a slightly different set of papers, both in searching in the databases and in inclusion/exclusion process.

We kept the gap between conducting searches in different sources less than one week, and finally updated the results in January 2010 to ensure capturing all studies published in 2009 (or at least entered into the databases before the end of 2009).

Some papers may have been missed due to application of constraints on the search strings in order to reduce the number of irrelevant papers found in the searches. We do not claim at collecting all relevant studies, but we included as many studies as possible. It should also be noted that although some studies may have been missed, there is no reason to believe that they would be distributed differently across the classifications than the papers included in the systematic map presented.

Since many empirical papers that we studied did not provide sufficient contextual details, we derived some data from the text (e.g. project size and duration). It has been impossible to judge the reported content separately, hence we trusted authors about what they reported on agile practices, distribution type, and the success of the project. It may have led to some unwanted inaccuracies in data extraction process.

In summary, we can claim that although the findings of similar studies may be slightly different from the findings of this research regarding numbers and figures; it will not change the patterns we have identified.

\section{CONCLUSIONS}

The current research literature on the application of different agile practices in GSE was summarized in this study. Further, the successful empirical studies were explored to investigate under which circumstances they have been efficiently practiced in software organizations.

\section{A. Growing Interest}

The applicability of agile practices in GSE is not yet well investigated. It is clear that several challenges are associated with combining them. However, an increasing number of publications, in particular experience reports, in last five years, indicates a growing interest in this area from software industry.

\section{B. Research Type}

The majority of the existing research literature is in the form of industrial experience reports. It reveals the need for conducting more evaluation research by which actual practices will be comprehensively examined. This type of research requires rigorous research methods and literature reviews, so one possible option could be close collaboration of industry and academia in this area. The research part can be done in academia while data has to be collected from real industrial cases.

\section{Repetitions}

We observed some repetitions in the content of the studies we explored. Similar problems are reported more than once in different articles [S74][S22]. It may indicate that previous research is not studied in software organizations or it is hard to interpret the context of different experiences. Another evidence for this conclusion is that industrial experience reports do not normally include the related work and do not reference literature. However, it requires further investigation to realize whether the academic materials such as textbooks or research papers are of interest for industry in this specific area.

\section{Corresponding Challenges}

There are not a sufficient number of studies analyzing the challenges of applying agile in GSE. Problems and challenges are documented in GSE or agile, while the combination is not well examined in real world situations. Some academic studies suggested that agile mitigates GSE challenges [S16][S42], whilst others believe they are contradictory in nature and it emphasizes the GSE challenges [S9]. Hence, we conclude that there is a need for in-depth studying of challenges and benefits of combining agile and GSE in the form of evaluation research.

\section{E. Contextual Information}

As mentioned previously, the contextual details for many empirical studies in this area is insufficient. Having this information assists researchers in examining the practical applicability of the reported cases for other settings. It demands researchers in this area to design and use a template for documenting the contextual information, which is not too detailed and not too abstract. We recommend practitioners and researchers to read guidelines presented by Petersen and Wohlin [10] and keep them in mind when writing their reports.

\section{F. Scaling up Agile}

There is not sufficient evidence to conclude that agile is efficiently applicable in large distributed projects. Although few studies have reported their experiences of large projects such as [S40][S41], the other contextual project factors are not clearly reported. 


\section{G. Modified Agile Practices}

In many studies that we reviewed, agile practices had been customized and a modified agile method was applied [S77]. The motivations for these adjustments were reported to be distribution type, overlapping working hours or other factors depending on the situational requirements of the project.

It highlights the need for further research in which the modifications are well studied in order to provide guidelines for practitioners on how to adapt the practices to their needs. In addition, the changes shall be compared to the original descriptions (e.g. agile manifesto) and determine the safe variance of the changes to remain agile, and of course efficient in software development. In other words, it shall be determined that how much change is allowed to be still recognized as practicing agile in GSE.

\section{H. Agile Applicability Framework}

This study pictured the current status of the studied area based on available peer-reviewed research literature, and the discussions in this section displayed the current improvement opportunities in the area.

In summary, the emergent need can be explained as developing a comprehensive framework that considers various factors from different perspectives when applying agile in GSE. It can be used as a basis for decision-making in early phases of software development, and assists project managers in estimating the risks, challenges, and benefits of using agile in (globally) distributed projects.

In the future, this systematic map will be complemented with a systematic review. Additional papers extracted from Scopus database (http://www.scopus.com) will be also investigated in order to provide as much input as possible for analyzing the current status of the area. These results will be used towards proposing such a comprehensive framework for agile applicability in GSE. Currently, we are working on developing a model in order to provide a unified concrete basis for judgments about accordance to agile values and principals in different organizational settings.

\section{ACKNOWLEDGMENT}

This work was partly funded by the Industrial Excellence Center EASE - Embedded Applications Software Engineering, (http://ease.cs.lth.se).

\section{REFERENCES}

[1] P. Abrahamsson, J. Warsta, M.T. Siponen, J. Ronkainen, "New Directions on Agile Methods: a Comparative Analysis", Proceedings of the 25th International Conference on Software Engineering, ACM Press, 2003, pp. 244-254.

[2] A. Abran, J.W. Moore, Guide to the Software Engineering Body of Knowledge (SWEBOK ${ }^{\circledR}$ ), IEEE Computer Society 2004 Guide, Angela Burgess, 2004.

[3] B. Boehm, R. Turner, "Management Challenges to Implement Agile Processes in Traditional Development Organizations", IEEE Software (22)5, 2005, pp. 30-39.

[4] S. Bowen, F. Maurer, "Process Support and Knowledge Management for Virtual Teams Doing Agile Software Development", Proceedings of the 26th Annual International Computer Software and Applications Conference, IEEE Computer Society Press, 2002, pp. 1118-1120.
[5] G. Corbitt, L.R. Gardiner, L.K. Wright, "A Comparison of Team Developmental Stages, Trust and Performance for Virtual versus Face-to-Face Teams", Proceedings of the 37th Hawaii International Conference on System Sciences, 2004.

[6] T. Dybå, T. Dingsøyr, "Empirical Studies of Agile Software Development: a Systematic Review", Journal of Information and Software Technology 50 (2008), 2008, pp. 833-859.

[7] J. Erickson, K. Lyytinen, K. Siau, "Agile Modeling, Agile Software Development, and Extreme Programming: the State of Research", Journal of Database Management 16 (4), 2005, pp. 88-100.

[8] B. Kitchenham, S. Charters, Guidelines for Performing Systematic Literature Reviews in Software Engineering, Technical Report EBSE2007-01, School of Computer Science and Mathematics, Keele University, 2007.

[9] K. Petersen, R. Feldt, S. Mujtaba, M. Mattsson, "Systematic Mapping Studies in Software Engineering", 12th International Conference on Evaluation and Assessment in Software Engineering, June 2008, pp. 71-80.

[10] K. Petersen, C. Wohlin, "Context in Industrial Software Engineering Research", 3rd International Symposiumm on Empirical Software Engineering and Measurement, 2009, pp. 401-404.

[11] R. Prikladnicki, J.L.N. Audy, D. Damian, T.C. de Oliveira, "Distributed Software Development: Practices and challenges in different business strategies of offshoring and onshoring", Proceedings of the IEEE International Conference on Global Software Engineering (ICGSE), 2007, pp. 262-274.

[12] H. Sharp, H. Robinson, "An Ethnographic Study of XP Practice", Journal of Empirical Software Engineering 9(4), 2004, pp. 353-375.

[13] D. Smite, C. Wohlin, R. Feldt, T. Gorschek, "Reporting Empirical Research in Global Software Engineering: a Classification Scheme", Proceedings of International Conference on Global Software Engineering, 2008, pp. 173-181.

[14] D. Smite, C. Wohlin, T. Gorschek, R. Feldt, "Empirical Evidence in Global Software Engineering: A Systematic Review", Journal of Empirical Software Engineering 15(1), 2010, pp. 91-118.

[15] R. Wieringa, N.A.M. Maiden, N.R. Mead, C. Rolland, "Requirements Engineering Paper Classification and Evaluation Criteria: a Proposal and a Discussion", Journal of Requir. Eng. 11(1), 2006, pp. 102-107.

[16] P.J. Ågerfalk, B. Fitzgerald, H. Holmström, B. Lings, B. Lundell, E. Ó Conchúir, "A Framework for Considering Opportunities and Threats in Distributed Software Development", Proceedings of International Workshop on Distributed Software Development, Austrian Computer Society, 2005, pp. 47-61.

\section{APPENDIX 1: DATA EXTRACTION FORM}

\section{General}

- Publication year: (1999-2009)

- Database: (ACM, IEEE, Inspec, Compendex, AIS)

- Number of authors $>=1$

- Authors' background: (industry, academic, unclear)

- Affiliations

- Countries

Agile Practices

- Main practice: (agile, scrum, XP, pair programming, lean)

- Sub-practices

- $\quad$ Agility level: (not all teams, all team, organization) GSE Settings

- Distributed type: (distributed team, virtual team, offshore, outsource, open source)

- Global: (yes, no, unclear)

- Number of sites $>=1$

- Countries Research Methodology

- $\quad$ Empirical: (yes, no, unclear) 
- Research type: (evaluation, validation, solution proposal, philosophical, personal experience, personal opinion)

- $\quad$ Research method: (qualitative, quantitative, mixed)

- Research sub-method: (single case study, multiple case study, experiment, literature review, etc)

- Means of data collection: (survey, questionnaire, interview, literature, etc)

- Means of analysis: (comparison, descriptive, measurement, classification, etc)

\section{Empirical Project Features}

- $\quad$ Size: (small, medium, large, unclear)

- Duration: (short, medium, long, unclear)

- Participants: (industry, students, unclear)

- Domain: (telecom, oil industry, web based, real time, embedded, etc)

- Knowledge area: (requirements engineering, design, development, testing, tools, project management, quality, etc)

- Successful: (yes, no, unclear) Results

- Contributions: (problem report, recommendations, lessons learned, tools, framework, etc)

\section{APPENDIX 2: INCLUDED STUDIES}

[S1] F. Abbattista, F. Calefato, D. Gendarmi, F. Lanubile, "Incorporating Social Software into Distributed Agile Development Environments", Automated Software Engineering - Workshops, 2008. ASE Workshops 2008. 23rd IEEE/ACM International Conference on, 2008, pp. 46-51.

[S2] S. Andrzeevski, "Experience Report 'Offshore XP for PDA Development"', AGILE 2007, 2007, pp. 376-381.

[S3] P.G. Armour, "Agile...and Offshore", Communications of the ACM 50(1), 2007, pp. 13-16.

[S4] D. Batra, "Modified Agile Practices for Outsourced Software Projects", Communications of the ACM 52(9), 2009, pp. 143-148.

[S5] S. Berczuk, "Back to Basics: The Role of Agile Principles in Success with an Distributed Scrum Team", AGILE 2007, 2007, pp. 382-388.

[S6] I. Bose, "Lessons Learned From Distributed Agile Software Projects: A Case-based Analysis", Communications of the Association for Information Systems 23(34), 2008.

[S7] S. Bowen, F. Maurer, "Process Support and Knowledge Management for Virtual Teams Doing Agile Software Development", ProceedingsIEEE Computer Society's International Computer Software and Applications Conference, 2002, pp. 1118-1120.

[S8] K. Braithwaite, T. Joyce, "Xp Expanded: Distributed Extreme Programming", 6th International Conference Extreme Programming and Agile Processes in Software Engineering, Lecture Notes in Computer Science, vol. 3556, 2005, pp. 180-8.

[S9] G. Canfora, A. Cimitile, G.A. Di Lucca, C.A. Visaggios, "How Distribution Affects the Success of Pair Programming", International Journal of Software Engineering and Knowledge Engineering 16 (2), 2006, pp. 293-313.

[S10]I. Chubov, D. Droujkov, "User Stories and Acceptance Tests as Negotiation Tools in Offshore Software Development", Lecture Notes in Computer Science, vol. 4536 LNCS, 2007, pp. 167-168.

[S11]B. Cohen, M. Thias, "The Failure of the Off-shore Experiment: a Case for Collocated Agile Teams", AGILE '09, 2009, pp. 251-256.

[S12]M. Cottmeyer, "The Good and Bad of Agile Offshore Development", AGILE '08, 2008, pp. 362-367.

[S13]M. Cristal, D. Wildt, R. Prikladnicki, "Usage of Scrum Practices within a Global Company", IEEE International Conference on Global Software Engineering, 2008, pp. 222-226.

[S14]A. Danait, "Agile Offshore Techniques - a Case Study", Proceedings Agile 2005, 2005, pp. 214-17.
[S15]B. Drummond, J. Unson, "Yahoo! Distributed Agile: Notes From The World Over", AGILE '08, 2008, pp. 315-321.

[S16]K. Dullemond, B. van Gameren, R. van Solingen, How Technological Support Can Enable Advantages of Agile Software Development in a GSE setting", Fourth IEEE International Conference on Global Software Engineering, 2009, pp. 143-152.

[S17]J. Eckstein, "Agile Development in the Face of Global Software Projects", Cutter IT J. (USA) 20(5), 2007, pp. 12-17.

[S18]M. Edwards, "Overhauling a Failed Project Using Out-of-the-box Scrum”, AGILE ’08, 2008, pp. 413-416.

[S19]M. Farmer, "Decisionspace Infrastructure: Agile Development in a Large, Distributed Team", Proceedings of the Agile Development Conference, 2004, pp. 95-99.

[S20]J. Fewell, "Growing PMI ${ }^{\circledR}$ Using Agile”, Agile Conference, 2009, pp. 356-360.

[S21]N.V. Flor, "Globally Distributed Software Development and Pair Programming", Communication of ACM 49(10), 2006, pp. 57-58.

[S22]F. Grossman, J. Bergin, D. Leip， S. Merritt， O. Gotel，"One XP Experience: Introducing Agile (XP) Software Development into a Culture That Is Willing But Not Ready", Proceedings of the 2004 conference of the Centre for Advanced Studies on Collaborative research (CASCON '04), IBM Press, 2004, pp. 242-254.

[S23]O. Hazzan, Y. Dubinsky, "Can Diversity in Global Software Development be Enhanced by Agile Software Development?", Proceedings of the 2006 international workshop on Global software development for the practitioner, 2006, pp. 58-61.

[S24]H. Holmstrom, B. Fitzgerald, P. Aagerfalk, E. Conchuir, "Agile Practices Reduce Distance in Global Software Development", Information Systems Management 23(3), 2006, pp. 7-18.

[S25]H. Holz, F. Maurer, "Knowledge Management Support for Distributed Agile Software Processes", 4th International Workshop Advances in Learning Software Organizations, Lecture Notes in Computer Science, vol. 2640, 2003, pp. 60-80.

[S26]E. Hossain, M. Ali Babar, J. Verner, "Towards a Framework for Using Agile Approaches in Global Software Development", Lecture Notes in Business Information Processing, vol. 32, 2009, pp. 126140.

[S27]E. Hossain, M. Babar, H. young Paik, "Using Scrum in Global Software Development: a Systematic Literature Review", Fourth IEEE International Conference on Global Software Engineering, 2009, pp. 175-184.

[S28]E. Hossain, M. Babar, H. young Paik, J. Verner, "Risk Identification and Mitigation Processes for Using Scrum in Global Software Development: a Conceptual Framework", Asia-Pacific Software Engineering Conference, 2009, pp. 457-464.

[S29]L. Hvatum, "Agile Practices and Distributed Teams", Cutter IT J. (USA) 20(5), 2007, pp. 6-11.

[S30]N. Jain, "Offshore Agile Maintenance”, AGILE 2006, 2006, pp. 327333.

[S31]M. Korkala, P. Abrahamsson, "Communication in Distributed Agile Development: a Case Study", 33rd Conference on Software Engineering and Advanced Applications, 2007, pp. 203-210.

[S32]A. Kornstadt, J. Sauer, "Mastering Dual-shore Development - the Tools and Materials Approach Adapted to Agile Offshoring", Lecture Notes in Computer Science, vol. 4716 LNCS, 2007, pp. 83-95.

[S33]C. Kussmaul, R. Jack, B. Sponsler, "Outsourcing and Offshoring with Agility: a Case Study", 4th Conference on Extreme Programming and Agile Methods, Lecture Notes in Comput. Sci., vol. 3134, 2004, pp. 147-54.

[S34]K. Kvam, R. Lie, D. Bakkelund, "Legacy System Exorcism by Pareto's Principle", Companion to the 20th annual conference on Object-oriented programming, systems, languages, and applications, 2005, pp. 250-256.

[S35]L. Layman, L. Williams, D. Damian, H. Bures, "Essential Communication Practices for Extreme Programming in a Global 
Software Development Team", Information and Software Technology 48(9), 2006, pp. 781-794.

[S36]D. Mak, P. Kruchten, "Nextmove: a Distributed Project Management Tool", Proceedings of the IASTED International Conference on Software Engineering, 2007, pp. 13-18.

[S37]D.K. Mak, P.B. Kruchten, "Task Coordination in an Agile Distributed Software Development Environment", Canadian Conference on Electrical and Computer Engineering, 2006, pp. 606-611.

[S38]A. Martin, R. Biddle, J. Noble, "When XP Met Outsourcing", 5th International Conference Extreme Programming and Agile Processes in Software Engineering, Lecture Notes in Comput. Sci., vol. 3092, 2004, pp. 51-9.

[S39]J. Mc Cormick, "Agile Phase I - the Pragmatic Case Study of Schneider National”, AGILE '05, 2005, pp. 212-213.

[S40]A. Miller, "A hundred days of continuous integration", AGILE '08, 2008, pp. 289-293.

[S41]J. Nielsen, D. McMunn, "The Agile Journey-Adopting XP in a Large Financial Services Organization", Lecture Notes in Computer Science, vol. 3556, 2005, pp. 28-37.

[S42]M. Nisar, T. Hameed, "Agile Methods Handling Offshore Software Development Issues", Proceedings of INMIC, 2004, pp. 417-422.

[S43]M. Paasivaara, S. Durasiewicz, C. Lassenius, "Distributed Agile Development: Using Scrum in a Large Project", International Conference on Global Software Engineering, 2008, pp. 87-95.

[S44]M. Paasivaara, S. Durasiewicz, C. Lassenius, "Using Scrum in Distributed Agile Development: A multiple Case Study", International Conference on Global Software Engineering, 2009, pp. 195-204

[S45]J.S. Persson, I. Aaen, L. Mathiassen, "Real-Time Control Mediation in Agile Distributed Software Development", AMCIS 2008 proceedings, Number 293. 2008.

[S46]R. Phalnikar, V. Deshpande, S. Joshi, "Applying Agile Principles for Distributed Software Development", International Conference on Advanced Computer Control, 2009, pp. 535-539.

[S47]C. Poole, "Distributed Product Development using Extreme Programming", 5th International Conference Extreme Programming and Agile Processes in Software Engineering, Lecture Notes in Comput. Sci., vol. 3092, 2004, pp. 60-7.

[S48]N. Ramasubbu, R.K. Balan, "The Impact of Process Choice in High Maturity Environments: an Empirical Analysis", 31st International Conference on Software Engineering, IEEE Computer Society, 2009, pp. 529-539.

[S49]B. Ramesh, L. Cao, K. Mohan, P. Xu, "Can Distributed Software Development be Agile?", Communications of the ACM 49(10), 2006, pp. 41-46.

[S50]M. Reeves, J. Zhu, "Moomba - a Collaborative Environment for Supporting Distributed Extreme Programming in Global Software Development", 5th International Conference Extreme Programming and Agile Processes in Software Engineering, Lecture Notes in Comput. Sci., vol. 3092, 2004, pp. 38-50.

[S51]J. Robarts, "Practical Considerations for Distributed Agile Projects", AGILE '08, 2008, pp. 327-332.

[S52]J. Rothman, "Agility in a Box [Project Scheduling Tool]", Soft. Dev. (USA) 12(3), 2004, pp. 25-7.

[S53]B. Roussev, R. Akella, "Agile Outsourcing Projects: Structure and Management", International Journal of e-Collaboration 2(4), 2006, pp. 37-52.

[S54]R. Sangwan, P. Laplante, "Test-driven Development in Large Projects", IT Professional 8(5), 2006, pp. 25-29.

[S55]T. Schummer, S. Lukosch, "Supporting the Social Practices of Distributed Pair Programming", Lecture Notes in Computer Science, vol. 5411 LNCS, 2008, pp. 83-98.

[S56]C. Sepulveda, "Agile Development and Remote Teams: Learning to Love the Phone", Proceedings of the Agile Development Conference, 2003, pp. 140-145.
[S57]B. Sheth, "Scrum 911! Using Scrum to Overhaul a Support Organization”, AGILE '09, 2009, pp. 74-78.

[S58]R. Sison, T. Yang, "Use of Agile Methods and Practices in the Philippines", 14th Asia-Pacific Software Engineering Conference, 2007, pp. 462-469.

[S59]H. Smits, G. Pshigoda, "Implementing Scrum in a Distributed Software Development Organization", AGILE '07, 2007, pp. 371375.

[S60]M. Summers, "Insights into an Agile Adventure with Offshore Partners", AGILE '08, 2008, pp. 333-338.

[S61]K. Sureshchandra, J. Shrinivasavadhani, "Adopting Agile in Distributed Development", International Conference on Global Software Engineering, 2008, pp. 217-221.

[S62]J. Sutherland， G. Schoonheim， N. Kumar， V. Pandey， S. Vishal, "Fully Distributed Scrum: Linear Scalability of Production between San Francisco and India”, AGILE '09, 2009, pp. 277-282.

[S63]J. Sutherland, G. Schoonheim, M. Rijk, "Fully Distributed Scrum: Replicating Local Productivity and Quality with Offshore Teams", 42nd Hawaii International Conference on System Sciences, 2009, pp. $1-8$.

[S64]J. Sutherland, A. Viktorov, J. Blount, N. Puntikov, "Distributed Scrum: Agile Project Management with Outsourced Development Teams", 40th Annual Hawaii International Conference on System Sciences, 2007, pp. 274a-274a.

[S65]P.S. Taylor, D. Greer, P. Sage, G. Coleman, K. McDaid, F. Keenan, "Do Agile GSD Experience Reports Help the Practitioner", Proceedings of the 2006 international workshop on Global software development for the practitioner, 2006, pp. 87-93.

[S66]E. Therrien, "Overcoming the Challenges of Building a Distributed Agile Organization", AGILE '08, 2008, pp. 368-372.

[S67]W.H.M. Theunissen, A. Boake, D.G. Kourie, "In Search of the Sweet Spot: Agile Open Collaborative Corporate Software Development", Proceedings of the 2005 annual research conference of the South African institute of computer scientists and information technologists on IT research in developing countries, 2005, pp. 268-277.

[S68]I. Turnu, M. Melis, A. Cau, M. Marchesi, A. Setzu, "Introducing TDD on a Free Libre Open Source Software Project: a Simulation Experiment", Proceedings of the 2004 workshop on Quantitative techniques for software agile process, 2004, pp. 59-65.

[S69]R. Urdangarin, P. Fernandes, A. Avritzer, D. Paulish, "Experiences with Agile Practices in the Global Studio Project", International Conference on Global Software Engineering, 2008, pp. 77-86.

[S70]E. Uy, N. Ioannou, "Growing and Sustaining an Offshore Scrum Engagement”, AGILE '08, 2008, pp. 345-350.

[S71]M. Vax, S. Michaud, Distributed agile: "Growing a Practice Together', AGILE '08, 2008, pp. 310-314.

[S72]P. Wagstrom, J. Herbsleb, "Dependency Forecasting in the Distributed Agile Organization", Communications of the ACM, 49(10), 2006, pp. 55-6.

[S73]D. Wahyudin, M. Heindl, B. Eckhard, A. Schatten, S. Biffl, "In-time Role-specific Notification as Formal Means to Balance Agile Practices in Global Software Development Settings", Central and East European Conference on Software Engineering Techniques, 2008, pp. 208-22.

[S74]W. Williams, M. Stout, "Colossal, Scattered, and Chaotic (Planning with a Large Distributed Team)”, AGILE '08, 2008, pp. 356-361.

[S75]B. Xu, X. Yang, Z. He, S. Maddineni, "Achieving High Quality in Outsourcing Reengineering Projects throughout Extreme Programming”, IEEE International Conference on Systems, Man and Cybernetics, vol. 3, 2004, pp. 2131-2136.

[S76]V. Yadav, M. Adya, D. Nath, V. Sridhar, "Investigating an "AgileRigid' Approach in Globally Distributed Requirements Analysis", PACIS 2007 Proceedings, 2007, Number 12.

[S77]C. Young, H. Terashima, "How Did We Adapt Agile Processes to Our Distributed Development”, AGILE '08, 2008, pp. 304-309. 